\title{
Risk factors affecting amputation in diabetic foot
}

\author{
Jun Ho Lee ${ }^{1}$, Ji Sung Yoon ${ }^{2}$, Hyoung Woo Lee ${ }^{2}$, Kyu Chang Won ${ }^{2}$, Jun Sung Moon ${ }^{2}$, Seung Min Chung ${ }^{3}$, \\ Yin Young Lee ${ }^{3}$
}

${ }^{1}$ Republic of Korea Army, Hwacheon, Korea

${ }^{2}$ Division of Endocrinology and Metabolism, Department of Internal Medicine, Yeungnam University College of Medicine, Daegu, Korea ${ }^{3}$ Division of Endocrinology and Metabolism, Department of Internal Medicine, Yeungnam University Hospital, Daegu, Korea

Received: March 4, 2020

Revised: April 9, 2020

Accepted: April 14, 2020

Corresponding author:

Ji Sung Yoon

Division of Endocrinology and

Metabolism, Department of Internal

Medicine, Yeungnam University

College of Medicine, 170

Hyunchung-ro, Nam-gu, Daegu

42415, Korea

Tel: +82-53-620-4049

Fax: +82-53-623-8006

E-mail: jsyoon9@ynu.ac.kr
Background: A diabetic foot is the most common cause of non-traumatic lower extremity amputations (LEA). The study seeks to assess the risk factors of amputation in patients with diabetic foot ulcers (DFU).

Methods: The study was conducted on 351 patients with DFUs from January 2010 to December 2018. Their demographic characteristics, disease history, laboratory data, ankle-brachial index, Wagner classification, osteomyelitis, sarcopenia index, and ulcer sizes were considered as variables to predict outcome. A chi-square test and multivariate logistic regression analysis were performed to test the relationship of the data gathered. Additionally, the subjects were divided into two groups based on their amputation surgery.

Results: Out of the 351 subjects, 170 required LEA. The mean age of the subjects was 61 years and the mean duration of diabetes was 15 years; there was no significant difference between the two groups in terms of these averages. Osteomyelitis (hazard ratio [HR], 6.164; 95\% confidence interval [Cl], 3.561-10.671), lesion on percutaneous transluminal angioplasty $(\mathrm{HR}, 2.494 ; 95 \% \mathrm{Cl}$, 1.087-5.721), estimated glomerular filtration rate (eGFR; $\mathrm{HR}, 0.99 ; 95 \% \mathrm{Cl}, 0.981-0.999$ ), ulcer size (HR, 1.247; 95\% Cl, 1.107-1.405), and forefoot ulcer location ( $\mathrm{HR}, 2.475 ; 95 \% \mathrm{Cl}$, 0.224-0.73) were associated with risk of amputation.

Conclusion: Osteomyelitis, peripheral artery disease, chronic kidney disease, ulcer size, and forefoot ulcer location were risk factors for amputation in diabetic foot patients. Further investigation would contribute to the establishment of a diabetic foot risk stratification system for Koreans, allowing for optimal individualized treatment.

Keywords: Amputation; Diabetic foot ulcer; Diabetes mellitus; Risk factors

\section{Introduction}

Diabetes is becoming increasingly prevalent worldwide due to aging, physical inactivity, westernized eating habits, population growth, and obesity; consequently, the incidences of diabetic feet are increasing. Diabetic patients are predicted to have a $25 \%$ probability of having at least one diabetic foot ulcer during their lifetime. Furthermore, a lower limb amputation due to a diabetic foot is carried out every 30 seconds worldwide, with rates being 30 to 40 times higher for diabetic patients than it is for individuals without the disease [1-3].

The cost of diabetic foot treatment accounts for approximately $25 \%$ of a diabetic patient's total hospital costs [4]. To reduce this burden, clinicians should focus on prevention as well as treatment of diabetic foot disease. Needless to say, amputation in patients with diabetic foot disease debilitates their ability to perform ev-

Copyright (C) 2020 Yeungnam University College of Medicine

This is an Open Access article distributed under the terms of the Creative Commons Attribution Non-Commercial License (http://creativecommons.org/licenses/by-nc/4.0/) which permits unrestricted non-commercial use, distribution, and reproduction in any medium, provided the original work is properly cited. 
eryday tasks, which negatively affects their quality of life ; therefore, knowledge of the risk factors of diabetic foot amputation is an important issue [5]. Minute observation and prophylactic action for patients at high risk of having a diabetic foot and early detection of foot complications could reduce the occurrence of ulcerations and amputations [6].

The development of a diabetic foot ulcer has multifactorial causes, and its principal factors include: diabetic peripheral neuropathy, infection, peripheral arterial disease, and socioeconomic status [7]. Moreover, various features such as age, smoking, foot deformities, poor glycemic control, ulcer size, hypertension, white blood cell count, and lipid abnormalities have also been reported as risk factors for diabetic foot amputation [8-11]. However, previous studies on the risk factors of diabetic feet indicated inconsistent results. Therefore, the aim of this study was to determine the risk factors of amputation in Korean diabetic foot patients who received standard treatment from one institution.

\section{Materials and methods}

\section{Subjects and data collection}

This study was approved by the Institutional Review Board (IRB) of the Yeungnam University Hospital (IRB No: 2019-03-040). It followed the Declaration of Helsinki on medical protocol and ethics. The patients' personal information was withheld from the researchers.

This case control study involved 425 subjects who were admitted to the Yeungnam University Hospital due to a diabetic foot from January 1, 2010 to December 31, 2018. Due to lack of data, 74 of the 425 subjects were excluded, leaving 351 valid subjects. They were divided into two groups based on their amputation surgery.

\section{Clinical information of patients}

Medical records, including admission notes, were examined to obtain information on the patients and laboratory results collected during the first day of admission. The independent variables were selected based on previous studies to determine the risk factors of diabetic feet and amputation. Amputation was defined as surgery, which goes beyond the toe level. Minor debridement of soft tissue was not considered as amputation surgery.

Hypertension was defined as the use of anti-hypertensive medication or previously documented diagnoses. Ankle-brachial index (ABI) was calculated by dividing the systolic blood pressure of the ankle divided by the systolic blood pressure of the upper arm of the affected side. The mean value of the ABI of the two groups were compared and subjects with $\mathrm{ABI}$ of less than 0.9 were found to be abnormal.

Diabetic peripheral polyneuropathy was identified through the consultation records of the neurology department, electrophysiology studies, the Semmes Weinstein monofilament test, clinical scores (i.e., the Michigan Neuropathy Screening Instrument questionnaire), and medical record reviews. Diabetic retinopathy was identified through ophthalmologic records, including any history of photocoagulation and vitrectomy. Coronary artery disease was defined as any history of myocardial infarction, unstable angina, percutaneous transluminal coronary angioplasty, or coronary artery bypass surgery. Stroke was defined as any history of cerebral infarction or transient cerebral ischemia. Chronic kidney disease was defined as the estimated glomerular filtration rate $<60 \mathrm{~mL} /$ $\mathrm{min} / 1.73 \mathrm{~m}^{2}$, which was calculated using the Modification of Diet in Renal Disease formula: $186 \times(\text { creatinine })^{-1.154} \times(\text { age })^{-0.203} \times(0.742$, if female). The sarcopenia index (SI) was used to estimate skeletal muscle mass and was derived from the formula serum creatinine value/cystatin C value [12].

Information of previous amputation history was based on orthopedic surgery and plastic surgery department records. The ulcer size was defined as the longest diameter, in centimeters, from one end of an ulcer margin to the other; this was assessed by an endocrinologist at admission. The Wagner classification categorized the diabetic foot ulcers based on the depth and the presence of osteomyelitis or gangrene. A grade ranging from 0 to 5 was assigned to pre-ulcerative lesions; partial superficial ulcers; extensions into tendons, ligaments, fascia, or joint capsules without osteomyelitis; deep ulcers with osteomyelitis; partial forefoot necrosis; and extensive foot gangrene, respectively [13]. The location of the foot ulcers was classified as forefoot, midfoot, and hindfoot. Percutaneous transluminal angioplasty (PTA) was assessed through records of the vascular department while osteomyelitis was assessed through scans such as an magnetic resonance imaging or 3-phase bone scan.

\section{Statistical analysis}

IBM SPSS version 20.0 (IBM Corp, Armonk, NY, USA) was used for statistical analysis and a Student $t$-test was conducted to compare the quantitative variables of the two groups. A chi-square test was used to analyze the categorial variables, and the risk factors for amputation were determined through stepwise multiple logistic regression analysis. All $p$-values less than 0.05 were considered statistically significant.

\section{Results}

The baseline characteristics of both groups are summarized in 
Table 1. Univariate analysis of subjects with or without amputation surgery

\begin{tabular}{|c|c|c|c|}
\hline Variable & Non-amputation group $(n=181)$ & Amputation group $(n=170)$ & $p$-value ${ }^{a}$ \\
\hline Age (yr) & $61.8 \pm 12.6$ & $62.4 \pm 10.9$ & 0.642 \\
\hline Body mass index $\left(\mathrm{kg} / \mathrm{m}^{2}\right)$ & $23.2 \pm 4.3$ & $23.0 \pm 3.6$ & 0.682 \\
\hline $\mathrm{SBP}(\mathrm{mmHg})$ & $130.7 \pm 17.5$ & $130.8 \pm 20.5$ & 0.959 \\
\hline $\mathrm{DBP}(\mathrm{mmHg})$ & $78.9 \pm 10.7$ & $77.1 \pm 12.8$ & 0.152 \\
\hline Length of hospitalization (day) & $51.1 \pm 54.9$ & $53.7 \pm 58.4$ & 0.660 \\
\hline Duration of diabetes (yr) & $15.4 \pm 9.5$ & $15.1 \pm 10.0$ & 0.821 \\
\hline Ankle-brachial index & $1.1 \pm 0.2$ & $1.0 \pm 0.3$ & 0.027 \\
\hline Size of ulcer $(\mathrm{cm})$ & $2.6 \pm 2.2$ & $3.6 \pm 2.6$ & 0.0001 \\
\hline Sarcopenia index & $101.7 \pm 101.5$ & $99.9 \pm 95.5$ & 0.861 \\
\hline $\mathrm{WBC}\left(\times 10^{3} / \mu \mathrm{L}\right)$ & $10.0 \pm 5.3$ & $11.2 \pm 6.4$ & 0.540 \\
\hline Hemoglobin (g/dL) & $11.3 \pm 2.0$ & $11.1 \pm 2.1$ & 0.526 \\
\hline $\mathrm{ESR}(\mathrm{mm} / \mathrm{hr})$ & $60.2 \pm 38.5$ & $66.8 \pm 36.3$ & 0.100 \\
\hline hsCRP (mg/L) & $6.7 \pm 9.5$ & $7.2 \pm 9.2$ & 0.603 \\
\hline Glycated hemoglobin (\%) & $8.9 \pm 2.2$ & $9.0 \pm 2.3$ & 0.565 \\
\hline Albumin (g/dL) & $3.5 \pm 0.8$ & $3.4 \pm 0.8$ & 0.293 \\
\hline Total cholesterol (mg/dL) & $158.0 \pm 50.7$ & $155.8 \pm 61.6$ & 0.730 \\
\hline Triglyceride (mg/dL) & $139.9 \pm 100.5$ & $161.6 \pm 172.0$ & 0.173 \\
\hline $\mathrm{HDL}-\mathrm{C}(\mathrm{mg} / \mathrm{dL})$ & $38.2 \pm 15.4$ & $33.8 \pm 16.1$ & 0.015 \\
\hline $\mathrm{LDL}-\mathrm{C}(\mathrm{mg} / \mathrm{dL})$ & $93.1 \pm 36.3$ & $90.5 \pm 41.1$ & 0.533 \\
\hline Blood urea nitrogen $(\mathrm{mg} / \mathrm{dL})$ & $23.2 \pm 14.7$ & $22.9 \pm 16.7$ & 0.843 \\
\hline Creatinine (mg/dL) & $2.0 \pm 4.3$ & $1.8 \pm 2.1$ & 0.569 \\
\hline eGFR $\left(\mathrm{mL} / \mathrm{min} / 1.73 \mathrm{~m}^{2}\right)$ & $68.7 \pm 29.0$ & $62.2 \pm 28.6$ & 0.035 \\
\hline $\mathrm{ACR}(\mathrm{mg} / \mathrm{mmol})$ & $761.5 \pm 1,690.5$ & $978.6 \pm 2,394.2$ & 0.531 \\
\hline Cystatin C (mg/dL) & $1.8 \pm 1.2$ & $2.0 \pm 1.49$ & 0.212 \\
\hline
\end{tabular}

Values are presented as mean \pm standard deviation.

SBP, systolic blood pressure; DBP, diastolic blood pressure; WBC, white blood cell; ESR, erythrocyte sedimentation rate; hsCRP, high-sensitivity C-reactive protein; HDL-C, high density lipoprotein cholesterol; LDL-C, low density lipoprotein cholesterol; eGFR, estimated glomerular filtration rate; ACR, albumin creatinine ratio.

a) $p$-value based on Student $t$-test.

Table 1 . The mean age of the subjects and the mean duration of diabetes was 61 and 15 years, respectively; there was no significant difference between the two groups. Among the amputation group, 119 subjects underwent surgery below the ankle, while 51 subjects had above-the-knee or below-the-knee amputations.

The amputation group generally had significantly lower high density lipoprotein cholesterol (HDL-C) and eGFR, but a larger ulcer size. The chi-square test indicated that the amputation group had a higher incidence of previous amputation history (Table 2). The use of statins, antiplatelet drugs, anti-hypertensive drugs, and insulin did not show any significant difference between the two groups.

Among the 351 subjects, 193 showed positive wound culture results. The most common pathogens were Staphylococcus aureus, followed by Pseudomonas aeruginosa, and Escherichia coli. The two groups did not exhibit any differences in terms of pathogens.

According to the Wagner classification, the chi-square test indicated a significant difference between the two groups $(p=0.0001)$, whereas the multivariate regression analysis did not (Table 2).

The non-amputation group consisted of 110, 61, and 10 subjects with forefoot, midfoot, and hindfoot ulcers, respectively. On the other hand, the amputation group had 129, 37, and four subjects with forefoot, midfoot, and hindfoot ulcers, respectively.

The ABI of 126 subjects were each obtained from the non-amputation and the amputation group and 26 and 46 of them had decreased ABI value, respectively. The chi-square test $(p=0.005)$ showed a significance between the two groups and the decreased $\mathrm{ABI}$ indicates an increased risk of amputation. Due to insufficient data, the $A B I$ was excluded from the regression analysis.

By means of multivariate regression analysis, the risk factors of amputation were identified as the presence of osteomyelitis of OR 6.164, a lesion on PTA of OR 2.494, a forefoot location of OR 2.475 , an ulcer size of OR 1.247, and a kidney function of OR 0.99 (Table 3). 
Table 2. Major risk factors of amputation

\begin{tabular}{|c|c|c|c|}
\hline Variable & Non-amputation $(n=181)$ & Amputation $(n=170)$ & $p$-value ${ }^{\text {a) }}$ \\
\hline Osteomyelitis & $77(42.5)$ & $142(83.5)$ & 0.0001 \\
\hline Lesion on percutaneous transluminal angioplasty & $10(5.5)$ & $30(17.6)$ & 0.0001 \\
\hline Diabetic peripheral polyneuropathy & $120(66.2)$ & $97(57.0)$ & 0.095 \\
\hline Chronic kidney disease $\left(\mathrm{eGFR}<60 \mathrm{~mL} / \mathrm{min} / 1.73 \mathrm{~m}^{2}\right)$ & $49(27.0)$ & $66(36.4)$ & 0.019 \\
\hline$A B I$ decreased $(A B I<0.9)$ & $26(n=126,20.6)$ & $46(n=126,36.5)$ & 0.005 \\
\hline Previous amputation history & $12(6.6)$ & $22(12.9)$ & 0.046 \\
\hline Hypertension & $113(62.4)$ & $103(60.6)$ & 0.723 \\
\hline Smoking & $79(43.6)$ & $82(48.2)$ & 0.358 \\
\hline Alcohol & $60(33.1)$ & $70(41.2)$ & 0.108 \\
\hline Wagner classification & & & 0.0001 \\
\hline Grade 0-1 & $72(39.7)$ & $30(17.6)$ & \\
\hline Garde 2 & $47(26.0)$ & $31(18.2)$ & \\
\hline Grade 3 & 36 (19.9) & $52(30.6)$ & \\
\hline Grade 4-5 & $26(14.4)$ & $57(33.5)$ & \\
\hline Ulcer location & & & 0.002 \\
\hline Forefoot & $110(60.8)$ & $129(75.9)$ & \\
\hline Midfoot & 61 (33.7) & 37 (21.8) & \\
\hline Hindfoot & $10(5.5)$ & $4(2.3)$ & \\
\hline
\end{tabular}

Values are presented as number (\%).

eGFR, estimated glomerular filtration rate; $\mathrm{ABI}$, ankle-brachial index.

${ }^{\text {a) }} p$-value based on chi-square test and Fisher exact test.

Table 3. Multivariate logistic regression analysis of diabetic foot amputation

\begin{tabular}{lccc}
\hline Variable & $p$-value & Odds ratio & $95 \% \mathrm{Cl}$ \\
\hline Osteomyelitis & 0.0001 & 6.164 & $3.561-10.671$ \\
Lesion on percutaneous transluminal angioplasty & 0.031 & 2.494 & $1.087-5.721$ \\
Previous amputation history & 0.089 & 2.092 & $0.894-4.894$ \\
Estimated glomerular filtration rate & 0.027 & 0.99 & $0.981-0.999$ \\
Alcohol & 0.065 & 1.637 & $0.97-2.763$ \\
Diabetic peripheral polyneuropathy & 0.039 & 0.57 & $0.334-0.973$ \\
Size of ulcer & 0.0001 & 1.247 & $1.107-1.405$ \\
Ulcer location & & & $0.224-0.73$ \\
$\quad$ Forefoot: midfoot & 0.003 & 2.475 & $0.072-1.158$ \\
$\quad$ Forefoot: hindfoot & 0.08 & 3.460 & \\
\hline
\end{tabular}

$\mathrm{Cl}$, confidence interval.

\section{Discussion}

While various risk factors of diabetic foot amputation may have been identified in previous research, this study found that osteomyelitis, ulcer size, chronic kidney disease, forefoot location, and peripheral arterial disease were associated with diabetic foot amputation. This diversity could be brought about by differences in the genetic profiles, treatment protocols, study designs, and cultural features of the study subjects.

Osteomyelitis is one of the most critical factors in diabetic foot treatment process. The removal of infected bones is crucial, as pri- or studies have suggested superior results from surgical therapy compared with medical therapy $[14,15]$. Moreover, surgical debridement was discovered to be necessary to control chronic bone infection because antimicrobial therapy alone showed low success rates in the event of osteomyelitis [16]. The presence of osteomyelitis was the most significant risk factor (OR, 6.164) for diabetic foot amputation in this study.

Arterial insufficiency causes an impairment of bloodstream (i.e., a shortage of nutrition, antibodies, and white blood cells), which leads to poor wound outcome. Claudication, diminished or absent lower extremity pulses, lower ankle blood pressure, $\mathrm{ABI}$, and trans- 
cutaneous oxygen pressure of the foot (foot TcPo2) were important risk factors of developing foot ulcer and amputation surgery, indicating peripheral artery disease in diabetic patients $[17,18]$. In this study, the presence of PTA lesions and lower ABIs were associated with an increased risk of amputation surgery. However, Sun et al. [19] demonstrated that a lower ABI was closely associated with risk of amputation only in Wagner grade 3 wounds, not in grades 2 or 4 . They assumed that a Wagner grade 4 grade wound would have catastrophic necrosis, which limits the influence of circulation; and vice versa for grade 2 wounds. This is why further comprehensive studies (i.e., with a subdivision of subjects with a classification of diabetic foot severity) focusing on the relationship between peripheral artery disease and prognosis are needed.

Ulcers located on the forefoot area were found to be a greater risk factor for diabetic foot amputation compared with midfoot or hindfoot located ulcers. Due to its distal position, it would be reasonable to assume that the forefoot area has the least sufficient blood supply in the foot region, resulting in a shortage of oxygen, white blood cell, and nutrition.

Several studies have shown that a large foot ulcer size and a high Wagner classification grade considerably increased the risk of amputation $[9,20]$. This study found that a higher Wagner classification grade and ulcer size were significantly associated with the risk of amputation through a chi-square test and regression analysis, respectively. This was in accordance with the findings of previous observational studies that more extensive wounds require more extensive surgical procedures such as amputation $[19,21]$.

Complications including diabetic microangiopathy often arise as a patient's diabetes progresses. Diabetic kidney disease is another complication, and it is known as a useful marker for the generalized vascular status of patients with diabetes. Additionally, patients with nephropathy are also prone to developing peripheral vascular disease [22]. Several studies have shown that the incidence of diabetic foot disease is more frequent among patients with albuminuria [23-25]. However, given the insufficient data on albuminuria, this study was unable to properly examine the relationship between albuminuria and amputation surgery.

Current dialysis for end-stage renal disease and chronic kidney disease were also identified as risk factors for diabetic feet and major amputation $[21,26]$. These results confirm that a lower eGFR and the presence of chronic kidney disease are associated with a higher risk of diabetic foot amputation.

Recently, sarcopenia has been demonstrated as an important risk factor of diabetic foot disease, which also influenced prognosis; however, the measurement of skeletal muscles entails expensive and complex imaging techniques [27]. Thus far, the gold standard of measuring skeletal muscle mass has been either body composition analysis or computed tomography. The SI is a novel biomarker for estimating muscle mass, which uses two molecules: cystatin $\mathrm{C}$, which originates from all nucleated cells and creatinine from skeletal muscle cells. The SI is significantly correlated with abdominal computed tomography and showed superior outcomes compared with serum creatinine on its own $[12,28,29]$. The study sought to assess the value of the SI as a marker to predict the prognosis of diabetic feet and compare it with other already established prognostic factors. In this study, the SI did not show statistically significant results. Since several studies have demonstrated the relationship between the SI and the prognosis of diabetic foot ulcers, this could be regarded as a limitation of the calculation formula.

The strength of this study is in its ethnic-specific design, wherein the chosen subjects share a common cultural, dietary lifestyle. Moreover, all of the subjects also underwent standard treatment protocols due to the single center-based recruitment process. These features greatly reduced biases present in previous studies that were brought about by the subjects' heterogeneous characteristics as well as the diverse treatment protocols among multicenters.

Conducting a larger scale study similar to this could lead to the establishment of a diabetic foot risk stratification system for Koreans.

The study has several limitations. Because this was a hospitalization-based single center design, a selection bias could not be excluded and the subjects may not have reflected the loco-regional population. So further multicenter studies are needed. This study was retrospective design, therefore independent variables were could not be fully assessed, and causality between each factors and outcome cannot be definitely established.

Osteomyelitis, a large ulcer size, nephropathy, forefoot location, and peripheral artery disease were identified as risk factors for amputation in hospitalized diabetic foot ulcer patients. Understanding their influence on amputation outcomes is necessary to develop risk stratification system, management, and treatment protocols for patients with diabetic feet. Through risk categorization, a multidisciplinary team for diabetic feet could receive timely assistance on decision-making (e.g., admission timing and invasive procedures), providing the best possible treatment for individualized patients.

\section{Acknowledgments}

\section{Conflicts of interest}

No potential conflict of interest relevant to this article was reported. 


\section{Author contributions}

Conceptualization: JHL, JSY, HWL, KCW, JSM, YYL; Data curation: JHL, SMC; Formal analysis: JSY, KCW, JSM, YYL; Methodology: JHL, JSY, JSM, YYL; Investigation: JHL, JSY, HWL, KCW, YYL; Project administration: JHL; Supervision: JSY, KCW, JSM; Resources: JSY; Visualization: JHL, SMC; Writing-original draft: JHL; Writing-review \& editing: JHL, JSM.

\section{ORCID}

Jun Ho Lee, https://orcid.org/0000-0002-5551-862X

Ji Sung Yoon, https://orcid.org/0000-0003-3091-3700

Hyoung Woo Lee, https://orcid.org/0000-0002-0773-1581

Kyu Chang Won, https://orcid.org/0000-0001-5945-3395

Jun Sung Moon, https://orcid.org/0000-0003-1569-3068

Seung Min Chung, https://orcid.org/0000-0003-3336-7557

Yin Young Lee, https://orcid.org/0000-0003-3684-8735

\section{References}

1. Boulton AJ, Vileikyte L, Ragnarson-Tennvall G, Apelqvist J. The global burden of diabetic foot disease. Lancet 2005;366:171924.

2. Singh N, Armstrong DG, Lipsky BA. Preventing foot ulcers in patients with diabetes. JAMA 2005;293:217-28.

3. Bakker K, Foster AV, van Houtum WH, Riley P; International Working Group on the Diabetic Foot. Diabetes and foot care: time to act. Brussels (BE): International Diabetes Federation and the International Working Group on the Diabetic Foot; 2005.

4. Songer TJ. The role of cost-effectiveness analysis and health insurance in diabetes care. Diabetes Res Clin Pract 2001;54(Suppl 1):S7-11.

5. Monteiro-Soares M, Boyko EJ, Ribeiro J, Ribeiro I, Dinis-Ribeiro M. Risk stratification systems for diabetic foot ulcers: a systematic review. Diabetologia 2011;54:1190-9.

6. Boulton AJ, Kirsner RS, Vileikyte L. Clinical practice: neuropathic diabetic foot ulcers. N Engl J Med 2004;351:48-55.

7. Markakis K, Bowling FL, Boulton AJ. The diabetic foot in 2015: an overview. Diabetes Metab Res Rev 2016;32(Suppl 1):169-78.

8. Fleischer AE, Wrobel JS, Leonards A, Berg S, Evans DP, Baron RL, et al. Post-treatment leukocytosis predicts an unfavorable clinical response in patients with moderate to severe diabetic foot infections. J Foot Ankle Surg 2011;50:541-6.

9. Oyibo SO, Jude EB, Tarawneh I, Nguyen HC, Armstrong DG, Harkless LB, et al. The effects of ulcer size and site, patient's age, sex and type and duration of diabetes on the outcome of diabet- ic foot ulcers. Diabet Med 2001;18:133-8.

10. Chaturvedi N, Stevens LK, Fuller JH, Lee ET, Lu M. Risk factors, ethnic differences and mortality associated with lower- extremity gangrene and amputation in diabetes. The WHO Multinational Study of Vascular Disease in Diabetes. Diabetologia 2001;44(Suppl 2):S65-71.

11. Resnick HE, Carter EA, Sosenko JM, Henly SJ, Fabsitz RR, Ness FK, et al. Incidence of lower-extremity amputation in American Indians: the Strong Heart Study. Diabetes Care 2004; 27:1885-91.

12. Kashani KB, Frazee EN, Kukralova L, Sarvottam K, Herasevich $\mathrm{V}$, Young PM, et al. Evaluating muscle mass by using markers of kidney function: development of the sarcopenia index. Crit Care Med 2017;45:e23-9.

13. Monteiro-Soares M, Martins-Mendes D, Vaz-Carneiro A, Sampaio S, Dinis-Ribeiro M. Classification systems for lower extremity amputation prediction in subjects with active diabetic foot ulcer: a systematic review and meta-analysis. Diabetes Metab Res Rev 2014;30:610-22.

14. Aragon-Sanchez FJ, Cabrera-Galvan JJ, Quintana-Marrero Y, Hernandez-Herrero MJ, Lazaro-Martinez JL, Garcia-Morales E, et al. Outcomes of surgical treatment of diabetic foot osteomyelitis: a series of 185 patients with histopathological confirmation of bone involvement. Diabetologia 2008;51:1962-70.

15. Ha Van G, Siney H, Danan JP, Sachon C, Grimaldi A. Treatment of osteomyelitis in the diabetic foot. Contribution of conservative surgery. Diabetes Care 1996;19:1257-60.

16. Jeffcoate WJ, Lipsky BA. Controversies in diagnosing and managing osteomyelitis of the foot in diabetes. Clin Infect Dis 2004; 39(Suppl 2):S115-22.

17. Boyko EJ, Ahroni JH, Stensel V, Forsberg RC, Davignon DR, Smith DG. A prospective study of risk factors for diabetic foot ulcer. The Seattle Diabetic Foot Study. Diabetes Care 1999;22: 1036-42.

18. Adler AI, Boyko EJ, Ahroni JH, Smith DG. Lower-extremity amputation in diabetes: the independent effects of peripheral vascular disease, sensory neuropathy, and foot ulcers. Diabetes Care 1999;22:1029-35.

19. Sun JH, Tsai JS, Huang CH, Lin CH, Yang HM, Chan YS, et al. Risk factors for lower extremity amputation in diabetic foot disease categorized by Wagner classification. Diabetes Res Clin Pract 2012;95:358-63.

20. Chuan F, Tang K, Jiang P, Zhou B, He X. Reliability and validity of the perfusion, extent, depth, infection and sensation (PEDIS) classification system and score in patients with diabetic foot ulcer. PLoS One 2015;10:e0124739.

21. Won SH, Chung CY, Park MS, Lee T, Sung KH, Lee SY, et al. 
Risk factors associated with amputation-free survival in patient with diabetic foot ulcers. Yonsei Med J 2014;55:1373-8.

22. Chuengsamarn S, Rattanamongkolgul S, Jirawatnotai S. Association between serum uric acid level and microalbuminuria to chronic vascular complications in Thai patients with type 2 diabetes. J Diabetes Complications 2014;28:124-9.

23. Al-Maskari F, El-Sadig M. Prevalence of risk factors for diabetic foot complications. BMC Fam Pract 2007;8:59.

24. Pradeepa R, Anjana RM, Unnikrishnan R, Ganesan A, Mohan V, Rema M. Risk factors for microvascular complications of diabetes among South Indian subjects with type 2 diabetes: the Chennai Urban Rural Epidemiology Study (CURES) Eye Study-5. Diabetes Technol Ther 2010;12:755-61.

25. Aragon-Sanchez J, Lazaro-Martinez JL, Garcia-Alvarez Y, Morales EG, Hernandez-Herrero MJ. Albuminuria is a predictive factor of in-hospital mortality in patients with diabetes admitted for foot disease. Diabetes Res Clin Pract 2014;104:e23-5.

26. Winkley K, Stahl D, Chalder T, Edmonds ME, Ismail K. Risk factors associated with adverse outcomes in a population-based prospective cohort study of people with their first diabetic foot ulcer.J Diabetes Complications 2007;21:341-9.

27. Cheng $\mathrm{Q}_{2} \mathrm{Hu}$ J, Yang P, Cao X, Deng X, Yang Q, et al. Sarcopenia is independently associated with diabetic foot disease. Sci Rep 2017;7:8372.

28. Shlipak MG, Mattes MD, Peralta CA. Update on cystatin C: incorporation into clinical practice. Am J Kidney Dis 2013;62: 595-603.

29. Kyhse-Andersen J, Schmidt C, Nordin G, Andersson B, Nilsson-Ehle P, Lindstrom V, et al. Serum cystatin C, determined by a rapid, automated particle-enhanced turbidimetric method, is a better marker than serum creatinine for glomerular filtration rate. Clin Chem 1994;40:1921-6. 